\title{
A Note on Cosmological Parameters and the Topology of the Universe
}

\author{
M. J. Rebouças \\ Centro Brasileiro de Pesquisas Físicas \\ Rua Dr. Xavier Sigaud 150 \\ 22290-180 Rio de Janeiro - RJ, Brazil \\ and J. S. Alcaniz \\ Observatório Nacional \\ Rua Gal. José Cristino 77 \\ 20921-400, Rio de Janeiro - RJ, Brazil
}

(Received on 28 November, 2005)

\begin{abstract}
Given the wealth of increasingly accurate cosmological observations, especially the recent results from the WMAP, and the development of methods and strategies in the search for cosmic topology, it is reasonable to expect that we should be able to detect the spatial topology of the Universe in the near future. Motivated by this, we examine to what extent a possible detection of a nontrivial topology of positively curved universe may be used to place constraints on the matter content of the Universe. We show through concrete examples that the knowledge of the spatial topology allows to place constraints on the density parameters associated to dark matter $\left(\Omega_{m}\right)$ and dark energy $\left(\Omega_{\Lambda}\right)$.
\end{abstract}

\section{INTRODUCTION}

Questions such as whether the Universe will expand forever or eventually re-collapse and end with a Big Crunch, and what its shape and size may be, are among the most fundamental challenges in cosmology. Regarding the former question, it is well known that the ultimate fate of the Universe is intrinsically associated with the nature of its dominant components. In the Friedmann-Lemaître-Robertson-Walker (FLRW) class of models, for instance, a universe that is dominated by a pressureless fluid (as, e.g., baryons and/or dark matter) or any kind of fluid with positive pressure (as radiation, for example) will expand forever if its spatial geometry is Euclidean or hyperbolic, or will eventually re-collapse if it is spherical. This predictable destiny for the Universe, however, may be completely modified if it is currently dominated by some sort of negative-pressure dark component, as indicated by a number of independent observational results (see, e.g., Ref. [1]). In this case, not only the dynamic but also the thermodynamic fate of the Universe may be completely different, with the possibility of an eternally expanding closed model [2], an increasingly hot expanding universe [3] or even a progressive rip-off of the large and small scale structure of matter ending with the occurrence of a curvature singularity, the so-called big smash [4].

The remaining questions, concerning the shape and size of our 3-dimensional world, go in turn beyond the scope of general relativity (GR), since they have an intrinsically topological nature. In this way, approaches or answers to these questions are ultimately associated with measurements of the global structure (topology) of the Universe and, as a local metric theory, GR cannot say much about it, leaving the global topology of the Universe undetermined[22]. Over the past few years, several aspects of the cosmic topology have become topical (see, e.g., the review articles Ref. [5]), given the wealth of increasingly accurate cosmological observations, especially the recent results from the Wilkinson Microwave Anisotropy Probe (WMAP) experiment [6], which have heightened the interest in the possibility of a universe with a nontrivial spatial topology[23].

A pertinent question the reader may ask at this point is whether the current values of cosmological density parameters, which help us to answer the above first question (associated with the ultimate fate of the Universe), can be constrained by a possible detection of the spatial topology of the Universe. Our primary objective here is to address this question by focusing our attention on possible topological constraints on the density parameters associated with the baryonic/dark matter $\left(\Omega_{m}\right)$ and dark energy $\left(\Omega_{\Lambda}\right)$. Motivated by the best fit value for the total energy density $\Omega_{\mathrm{T}} \equiv \Omega_{m}+\Omega_{\Lambda}=1.02 \pm 0.02$ $(1 \sigma$ level) reported by WMAP team [8], which includes a positively curved universe as a realistic possibility, we shall consider globally homogeneous spherical manifolds, some of which account for the suppression of power at large scales observed by WMAP [8], and also fits the WMAP temperature two-point correlation function $[11,12]$. To this end, in the next section we present our basic context and prerequisites, while in the last section we discuss our main results and present some concluding remarks.

\section{BASIC CONTEXT AND PREREQUISITES}

Within the framework of standard cosmology, the universe is described by a space-time manifold $\mathcal{M}_{4}=\mathbb{R} \times M$ with a locally homogeneous and isotropic Robertson-Walker (RW) metric

$$
d s^{2}=-d t^{2}+a^{2}(t)\left[d \chi^{2}+f^{2}(\chi)\left(d \theta^{2}+\sin ^{2} \theta d \phi^{2}\right)\right],
$$

where $f(\chi)=(\chi, \sin \chi$, or $\sinh \chi)$ depends on the sign of the constant spatial curvature $(k=0,1,-1$, respectively). The $3-$ space $M$ is usually taken to be one of the following simplyconnected spaces: Euclidean $\mathbb{R}^{3}$, spherical $\mathbb{S}^{3}$, or hyperbolic $\mathbb{H}^{3}$. However, given that the simple-connectedness of our space $M$ has not been established, our 3-space may equally well be any one of the possible quotient manifolds $M=\widetilde{M} / \Gamma$, 
where $\Gamma$ is a fixed point-free group of isometries of the covering space $\widetilde{M}=\left(\mathbb{R}^{3}, \mathbb{S}^{3}, \mathbb{H}^{3}\right)$. Thus, for example, in a universe whose geometry of the spatial section is Euclidean $(k=0)$, besides $\mathbb{E}^{3}$ there are 6 classes of topologically distinct compact orientable 3 -spaces $M$ that admits this geometry, while for universes with either spherical $(k=1)$ and hyperbolic $(k=$ $-1)$ spatial geometries there is an infinite number of topologically non-homeomorphic (inequivalent) manifolds with nontrivial topology that can be endowed with these geometries.

Quotient manifolds are compact in three independent directions, or compact in two or at least one independent direction. In compact manifolds, any two given points may be joined by more than one geodesic. Since the radiation emitted by cosmic sources follows geodesics, the immediate observational consequence of a nontrivial detectable spatial topology of $M$ is that the sky may show multiple images of radiating sources: cosmic objects or specific correlated spots of the cosmic microwave background radiation (CMBR). At very large scales, the existence of these multiple images (or pattern repetitions) is a physical effect that can be used to probe the 3space topology. In this work, we use the so-called "circlesin-the-sky" method (for cosmic crystallographic methods see, e.g., Refs. [13]), which relies on multiple copies of correlated circles in the CMBR maps [15], whose existence is clear from the following reasoning: In a space with a detectable nontrivial topology, the last scattering sphere (LSS) intersects some of its topological images along pairs of circles of equal radii, centered at different points on the LSS, with the same distribution of temperature fluctuations, $\delta T$. Since the mapping from the LSS to the night sky sphere preserves circles [16], these pairs of matching circles will be inprinted on the CMBR temperature fluctuations sky maps regardless of the background geometry and detectable topology. As a consequence, to observationally probe a nontrivial topology on the available largest scale, one should scrutinize the full-sky CMB maps in order to extract the correlated circles, whose angular radii and relative position of their centers can be used to determine the topology of the universe. Thus, a nontrivial topology of the space section of the universe may be observed, and can be probed through the circles-in-the-sky for all locally homogeneous and isotropic universes with no assumption on the cosmological density parameters.

Let us now state our basic cosmological assumptions and fix some notation. In addition to the RW metric (1), we assume that the current matter content of the Universe is well approximated by Cold Dark Matter (CDM) of density $\rho_{m}$ plus a cosmological constant $\Lambda$. In this standard $\Lambda \mathrm{CDM}$ context, for nonflat spaces the scale factor $a(t)$ can be identified with the curvature radius of the spatial section of the universe at time $t=t_{0}$, which is given by

$$
a_{0}^{2}=\frac{k}{H_{0}^{2}\left(\Omega_{\mathrm{T}}-1\right)},
$$

where here and in what follows the subscript 0 denotes evaluation at present time $t_{0}, H_{0}$ is the Hubble constant, and $\Omega_{\mathrm{T}}$ is the total density at $t=t_{0}$. In this way, for nonflat spaces the distance $\chi$ of any point with coordinates $(\chi, \theta, \phi)$ to the origin (in the covering space) in units of the curvature radius, $a_{0}=a\left(t_{0}\right)$, reduces to

$$
\chi=\sqrt{\left|\Omega_{k}\right|} \int_{1}^{1+z} \frac{d x}{\sqrt{x^{3} \Omega_{m 0}+x^{2} \Omega_{k}+\Omega_{\Lambda 0}}},
$$

where $x=1+z$ is an integration variable, and $\Omega_{k}=1-\Omega_{\mathrm{T}}$. Throughout this paper we shall measure the lengths in unit of curvature radius $a_{0}$.

A typical characteristic length of nonflat manifolds $M$, which we shall use in this paper, is the so-called injectivity radius $r_{i n j}$, which is defined as the radius of the smallest sphere 'inscribable' in $M$. An important mathematical result is that $r_{i n j}$, expressed in terms of the curvature radius, is a constant (topological invariant) for any given spherical and hyperbolic manifolds.

In this work we shall focus our attention in globally homogeneous spherical manifolds, as presented in Table I (see also its caption for more details). These manifolds satisfy a topological principle of homogeneity, in the sense that all points in $M$ are topologically equivalent.

\begin{tabular}{|cccc|}
\hline Name & Covering Group $\Gamma$ & Order of $\Gamma$ & $r_{i n j}$ \\
\hline \hline$Z_{n}$ & Cyclic $Z_{n}$ & $n$ & $\pi / n$ \\
$\mathcal{D}_{m}$ & Binary dihedral $D_{m}^{*}$ & $4 m$ & $\pi / 2 m$ \\
$\mathcal{T}$ & Binary tetrahedral $T^{*}$ & 24 & $\pi / 6$ \\
$O$ & Binary octahedral $O^{*}$ & 48 & $\pi / 8$ \\
$\mathcal{D}$ & Binary icosahedral $I^{*}$ & 120 & $\pi / 10$ \\
\hline
\end{tabular}

TABLE I: The globally homogeneous spherical manifolds are of the form $M=\mathbb{S}^{3} / \Gamma$. The first column gives the name we use for the manifolds. The second column displays the covering groups $\Gamma$. Finally, the remaining columns present the order of the group $\Gamma$ and the injectivity radius $r_{i n j}$. The cyclic and binary dihedral cases actually constitute families of manifolds, whose members are given by the different values of the integers $n$ and $m$. The order of $\Gamma$ gives the number of fundamental polyhedra needed to fulfill the whole covering space $\mathbb{S}^{3}$. Thus, for example, for the manifold $\mathcal{D}$ which is the the well-known Poincaré dodecahedral space, the fundamental polyhedron is a regular spherical dodecahedron, 120 of which tile the 3-sphere into identical cells that are copies of the FP.

\section{MAIN RESULTS AND FINAL REMARKS}

To investigate the extent to which a possible detection of a nontrival topology may place constraints on the cosmological density parameters, we consider here the globally homogeneous spherical manifolds. In these 3-spaces the number of pairs of matching circles depends on the ratio of the injectivity radius $r_{i n j}$ to the radius $\chi_{l s s}$ of LSS, which in turn depends on the density parameters (see Ref. [12] for examples of specific estimates of this number regarding $\mathcal{T}, O$ and $\mathcal{D}$ ). Nevertheless, if the topology of a globally homogeneous spherical manifold is detectable[24] the correlated pairs will be antipodal, i.e. the centers of correlated circles are separated by $180^{\circ}$, as shown in Figure 1.

Clearly the distance between the centers of each pair of the first correlated circles is twice the injectivity radius $r_{i n j}$. 


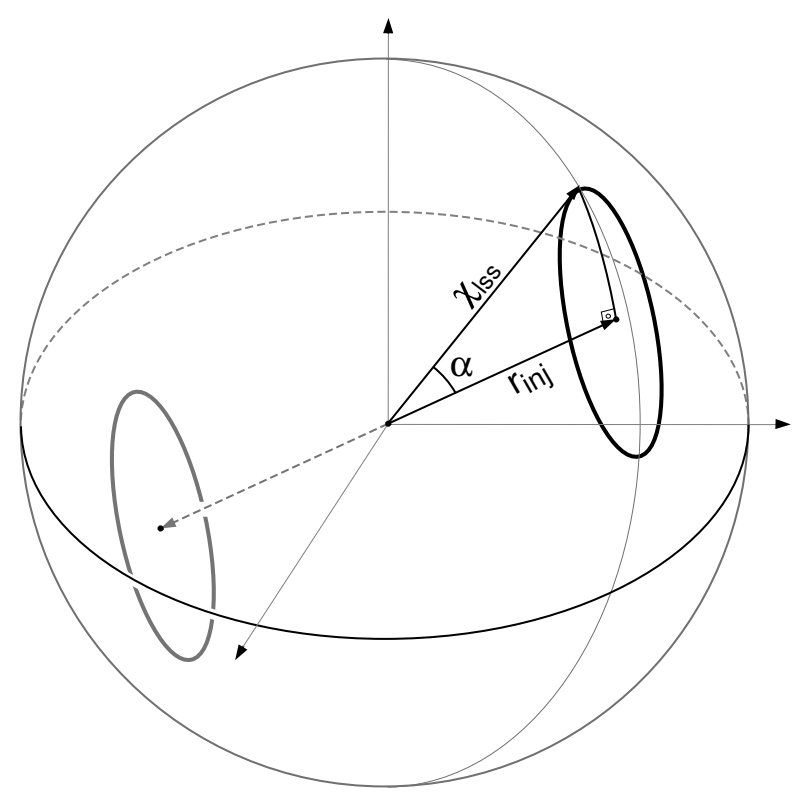

FIG. 1: A schematic illustration of two antipodal matching circles in the sphere of last scattering. These pair of circles occurs in all globally homogeneous positively curved manifolds with a detectable nontrivial topology. The relation between the angular radius $\alpha$ and the angular sides $r_{i n j}$ and $\chi_{l s s}$ is given $\cos \alpha=\tan r_{i n j} \cot \chi_{l s s}$.

Now, a straightforward use of known trigonometric rules to the right-angled spherical triangle shown in Figure 1 yields a relation between the angular radius $\alpha$ and the angular sides $r_{i n j}$ and radius $\chi_{l s s}$ of the last scattering sphere, namely

$$
\cos \alpha=\frac{\tan r_{i n j}}{\tan \chi_{l s s}}
$$

where $r_{i n j}$ is a topological invariant, whose values are given in Table I, and the distance $\chi_{l s s}$ of the last scattering surface to the origin in units of the curvature radius is given by (3) with $z_{l s s}=1089$ [8].

Equations (4) along with (3) give the relations between the angular radius $\alpha$ and the cosmological density parameters $\Omega_{\Lambda}$ and $\Omega_{m}$, and thus can be used to set bounds on these parameters. To quantify this we proceed in the following way. Firstly, as an example, we assume the angular radius $\alpha=50^{\circ}$. Secondly, since the measurements of the radius $\alpha$ unavoidably involve observational uncertainties, in order to obtain very conservative results we take $\delta \alpha \simeq 6^{\circ}$.[25]

In order to study the effect of the cosmic topology on the density parameters $\Omega_{m}$ and $\Omega_{\Lambda}$, we consider the binary tetrahedral $\mathcal{T}$ and the binary octahedral $O$ spatial topologies (see Table I), to reanalyze with these two topological priors the constraints on these parameters that arise from the so-called gold sample of 157 SNe Ia, as compiled by Riess et al. [19], along with the latest Chandra measurements of the X-ray gas mass fraction in $26 \mathrm{X}$-ray luminous, dynamically relaxed galaxy clusters (spanning the redshift range $0.07<z<0.9$ ) as provided by Allen et al. [20] (see also [21] for details on $\mathrm{SNe}$ Ia and X-ray statistics). The $\mathcal{T}$ and $O$ spatial topology is added to the conventional $\mathrm{SNe}$ Ia plus clusters data analysis as a Gaussian prior on the value of $\chi_{l s s}$, which can be easily obtained from an elementary combination of (4) and (3). In other words, the contribution of the topology to $\chi^{2}$ is a term of the form $\chi_{\text {topology }}^{2}=\left(\chi_{l s s}^{\mathrm{Obs}}-\chi_{l s s}^{\mathrm{Th}}\right)^{2} /\left(\delta \chi_{l s s}\right)^{2}$.

Figures $2 \mathrm{~b}$ and $2 \mathrm{c}$ (central and right panels) show the results of our statistical analysis. Confidence regions $-68.3 \%$ and $95.4 \%$ confidence limits (c.1.) - in the parametric space $\Omega_{m}-\Omega_{\Lambda}$ are displayed for the above described combination of observational data. For the sake of comparison, we also show in Fig. 2a the $\Omega_{m}-\Omega_{\Lambda}$ plane for the conventional SNe Ia plus Galaxy Clusters analysis, i.e., the one without the above cosmic topology assumption. By comparing both analyses, it is clear that a nontrivial space topology reduces considerably the parametric space region allowed by the current observational data, and also breaks some degeneracies arising from the current $\mathrm{SNe}$ Ia and X-ray gas mass fraction measurements. At $95.4 \%$ c.l. our $\mathrm{SNe} \mathrm{Ia}+\mathrm{X}$-ray+Topology analysis provides $\Omega_{m}=0.31 \pm 0.05$ and $\Omega_{\Lambda}=0.82 \pm 0.05$ (binary octahedral $O^{*}$ ) and $\Omega_{m}=0.32 \pm 0.06$ and $\Omega_{\Lambda}=0.89 \pm 0.06$ (binary tetrahedral $T^{*}$ ).

Concerning the above analysis it is worth emphasizing three important aspects. First, that the best-fit values depend weakly on the value used for radius $\alpha$ of the circle. Second, the uncertainty $\delta \alpha$ alters predominantly the area corresponding to the confidence regions, without having a significant effect on the best-fit values. Third, we also notice that there is a topological degeneracy in that the same the best fits and arise from either $Z_{6}$ or $\mathcal{D}_{3}$ spatial topology. Similarly, $O, Z_{8}$ and $\mathcal{D}_{3}$ give rise to identical bounds on the density parameters. This kind of topological degeneracy passed unnoticed in Refs. [18].

Finally, we emphasize that given the wealth of increasingly accurate cosmological observations, especially the recent results from the WMAP, and the development of methods and strategies in the search for cosmic topology, it is reasonable to expect that we should be able to detect it. Besides it importance as a major scientific achievement, we have shown through concrete examples that the knowledge of the spatial topology allows to place constraints on the density parameters associated to dark matter $\left(\Omega_{m}\right)$ and dark energy $\left(\Omega_{\Lambda}\right)$.

\section{Acknowledgements}

We thank CNPq for the grants under which this work was carried out. We also thank A.F.F. Teixeira for the reading of the manuscript and indication of relevant misprints and omissions.
[1] V. Sahni and A. Starobinsky, Int. J. Mod. Phys. D 9, 373 (2000); J.E. Peebles and B. Ratra, Rev. Mod. Phys. 75, 559 (2003); T.
Padmanabhan, Phys. Rep. 380, 235 (2003); J.A.S. Lima, Braz. 

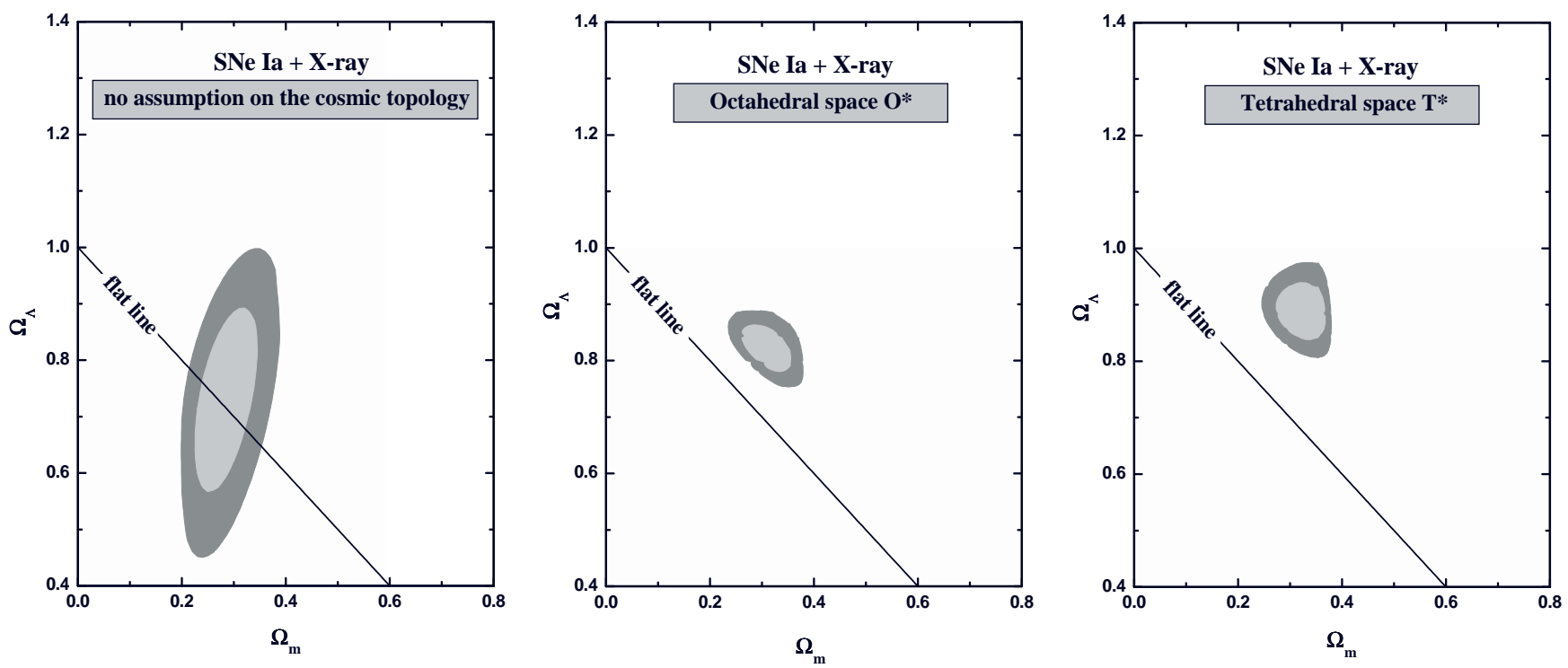

FIG. 2: The results of our statistical analyses. The panels show confidence regions (68.3\% and $95.4 \%$ c.1.) in the $\Omega_{m}-\Omega_{\Lambda}$ plane from the latest Chandra measurements of the X-ray gas mass fraction in 26 galaxy clusters $(0.07<z<0.9)$ plus determinations of the baryon density parameter, measurements of the Hubble parameter and the gold sample of 157 SNe Ia. Left Panel: The conventional SNe Ia plus X-ray analysis shown here for the sake of comparison. Central Panel: A binary octahedral space topology is assumed as the global topology of the Universe. The best fit values for the matter and vacuum density parameters are, respectively, $\Omega_{m}=0.31 \pm 0.05$ and $\Omega_{\Lambda}=0.82 \pm 0.05$ $\left(\Omega_{\mathrm{T}}=1.13 \pm 0.07\right)$ at $95.4 \%$ c.l.. Right Panel: A binary tetrahedral spatial topology is assumed. At $95.4 \%$ c.l., the best fit values for the matter and vacuum density parameters are $\Omega_{m}=0.32 \pm 0.06, \Omega_{\Lambda}=0.89 \pm 0.06$, and $\Omega_{\mathrm{T}}=1.21 \pm 0.08$.

J. Phys. 34, 194 (2004).

[2] J.E Felten and R. Isaacman, Rev. Mod. Phys. 58, 689 (1986).

[3] J.A.S. Lima and J.S. Alcaniz, Phys. Lett. B 600, 191 (2004).

[4] R.R. Caldwell, M. Kamionkowski, N.N. Weinberg, Phys. Rev. Lett. 91, 071301 (2003); S. Nesseris, L. Perivolaropoulos, Phys. Rev. D 70, 123529 (2004).

[5] M. Lachièze-Rey and J.-P. Luminet, Phys. Rep. 254, 135 (1995); G.D. Starkman, Class. Quantum Grav. 15, 2529 (1998); J. Levin, Phys. Rep. 365, 251 (2002); M.J. Rebouças and G.I. Gomero, Braz. J. Phys. 34, 1358 (2004).

[6] C.L. Bennett et al. , Astrophys. J. 583, 1 (2003); G. Hinshaw et al. , Astrophys. J. Suppl. 148, 135 (2003); C.L. Bennett et al. , Astrophys. J. Suppl. 148, 1 (2003).

[7] E. Komatsu et al. , Astrophys. J. Suppl. 148, 119 (2003); H.K. Eriksen, F.K. Hansen, A.J. Banday, K.M. Gorski, and P.B. Lilje, Astrophys. J. 605, 14 (2004); C.J. Copi, D. Huterer, and G.D. Starkman, Phys. Rev. D 70, 043515 (2004).

[8] D.N. Spergel et al., Astrophys. J. Suppl. 148, 175 (2003).

[9] M. Tegmark, A. de Oliveira-Costa, and A.J.S. Hamilton, Phys. Rev. D 68, 123523 (2003); A. de Oliveira-Costa, M. Tegmark, M. Zaldarriaga, and A. Hamilton, Phys. Rev. D 69, 063516 (2004); J.R. Weeks, astro-ph/0412231; P. Bielewicz, H.K. Eriksen, A.J. Banday, and K.M. Gorski, and P.B. Lilje, astroph/0507186; K. Land and J. Magueijo, Phys. Rev. Lett. 95, 071301 (2005). A. Bernui, B. Mota, M.J. Rebouças, and R. Tavakol, astro-ph/0511666; K. Land and J. Magueijo, Mon. Not. Roy. Astron. Soc. 357, 994 (2005).

[10] J.-P. Luminet, J. Weeks, A. Riazuelo, R. Lehoucq, and J.-P. Uzan, Nature 425, 593 (2003); N.J. Cornish, D.N. Spergel, G.D. Starkman, and E. Komatsu, Phys. Rev. Lett. 92, 201302 (2004); J. Gundermann, astro-ph/0503014; B.F. Roukema, B. Lew, M. Cechowska, A. Marecki, and S. Bajtlik, Astron. Astro- phys. 423, 821 (2004).

[11] R. Aurich, S. Lustig, and F. Steiner, Class. Quant. Grav. 22, 2061 (2005); R. Aurich, S. Lustig, and F. Steiner, Class. Quantum Grav. 22, 3443 (2005).

[12] R. Aurich, S. Lustig, and F. Steiner, astro-ph/0510847.

[13] R. Lehoucq, M. Lachièze-Rey, and J.-P Luminet, Astron. Astrophys. 313, 339 (1996); B.F. Roukema and A. Edge, Mon. Not. Roy. Astron. Soc. 292, 105 (1997); B.F. Roukema, Class. Quantum Grav. 15, 2645 (1998); R. Lehoucq, J.-P Luminet, and J.-P. Uzan, Astron. Astrophys. 344, 735 (1999); H.V. Fagundes and E. Gausmann, Phys. Lett. A 238, 235 (1998); H.V. Fagundes and E. Gausmann, Phys. Lett. A 261, 235 (1999); J.-P. Uzan, R. Lehoucq, and J.-P. Luminet, Astron. Astrophys. 351, 766 (1999); G.I. Gomero, M.J. Rebouças, and A.F.F. Teixeira, Int. J. Mod. Phys. D 9, 687 (2000); R. Lehoucq, J.-P. Uzan, and J.-P Luminet, Astron. Astrophys. 363, 1 (2000); G.I. Gomero, M.J. Rebouças, and A.F.F. Teixeira, Phys. Lett. A 275, 355 (2000); G.I. Gomero, M.J. Rebouças, and A.F.F. Teixeira, Class. Quantum Grav. 18, 1885 (2001); G.I. Gomero, A.F.F. Teixeira, M.J. Rebouças, and A. Bernui, Int. J. Mod. Phys. D 11, 869 (2002); A. Marecki, B. Roukema, and S. Bajtlik, Astron. Astrophys. 435, 427 (2005).

[14] C.J. Copi, D. Huterer, D.J. Schwarz, and G.D. Starkman, astro$\mathrm{ph} / 0508047$.

[15] N.J. Cornish, D. Spergel, and G. Starkman, Class. Quantum Grav. 15, 2657 (1998).

[16] R. Penrose, Proc. Cambridge Philos. Soc. 55, 137 (1959).

[17] G.I. Gomero, M.J. Rebouças, and R. Tavakol, Class. Quantum Grav. 18, 4461 (2001); G.I. Gomero, M.J. Rebouças, and R. Tavakol, Int. J. Mod. Phys. A 17, 4261 (2002); J.R. Weeks, R. Lehoucq, and J.-P. Uzan, Class. Quantum Grav. 20, 1529 (2003); J.R. Weeks, Mod. Phys. Lett. A 18, 2099 (2003); G.I. 
Gomero and M.J. Rebouças, Phys. Lett. A 311, 319 (2003); B. Mota, G.I. Gomero, M. J. Rebouças and R. Tavakol, Class. Quantum Grav. 21, 3361 (2004).

[18] M.J. Rebouças, J.S. Alcaniz, B. Mota, and M. Makler, astroph/0511007; M.J. Rebouças and J.S. Alcaniz, submitted for publication (2005)

[19] A.G. Riess et al., Astrophys. J. 607, 665 (2004).

[20] S.W. Allen, R.W. Schmidt, H. Ebeling, A.C. Fabian, and L. van Speybroeck, Mon. Not. Roy. Astron. Soc. 353, 457 (2004).

[21] J.A.S. Lima, J.V. Cunha, and J.S. Alcaniz, Phys. Rev. D 68, 023510 (2003); J.S. Alcaniz and Z.-H. Zhu, Phys. Rev. D 71, 083513 (2005); D. Rapetti, S.W. Allen, and J. Weller, Mon. Not. Roy. Astron. Soc. 360, 546 (2005).

[22] In this paper, in line with the usage in the literature, by topology of the universe we mean the topology of its spatial section.
[23] For instance, the reported non-Gaussianity in cosmic microwave background (CMB) maps [7] along with the small power of large-angle fluctuations [8], and some features in the power spectrum [7-9] are all large-scale anomalies which have been suggested as potential indications of a universe with nontrivial topology [10, 11]. See Ref. [14] for a detailed discussion on these anomalies.

[24] The extent to which a nontrivial topology may or may not be detected has been discussed in references [17].

[25] These values are taken as typical and for the sake of comparison with the results of Refs. [18]. We note, however, that our general conclusions hold regardless of the precise value for $\alpha$ and its uncertainty. 